\title{
Observation of single-diffractive $W$ production with CMS: a feasibility study
}

\author{
A. Vilela Pereira, on behalf of the CMS Collaboration \\ Università degli Studi di Torino \& INFN Torino \\ Via Pietro Giuria 1, 10125 Torino, Italy
}

\begin{abstract}
We present a study of single-diffractive $W$-boson production in $p p$ collisions at $\sqrt{s}=$ $14 \mathrm{TeV}, p p \rightarrow X p$ with $X$ including a $W$ boson, with the CMS detector. We discuss the feasibility of observing this process with an integrated effective luminosity for single interactions of $100 \mathrm{pb}^{-1}$.
\end{abstract}

\section{Introduction}

In the present paper, the single-diffractive (SD) reaction $p p \rightarrow X p$ is studied, in which $X$ includes a $W$ boson (Fig. 11). The $W \rightarrow \mu \nu$ decay mode is considered. This reaction is sensitive to the diffractive structure function of the proton and to the rapidity gap survival probability 2. This process has been studied at the Tevatron, where the ratio of the yields for SD and inclusive $W$ production has been measured to be approximately $1 \%$, 4 ,

The aim of this analysis is to demonstrate the feasibility of observing SD $W$ production at CMS given an integrated effective luminosity for single interactions of $100 \mathrm{pb}^{-1}$; this effective luminosity will be lower than the integrated delivered luminosity and will depend on the machine conditions.

The CMS apparatus is described in detail elsewhere [5]. Two experimental scenarios are considered here. In the first, no forward detectors beyond the CMS forward calorimeter HF, which covers the region in pseudo-rapidity of $3<|\eta|<5$, are assumed. In the second, additional coverage at $-6.6<\eta<-5.2$ is assumed by means of

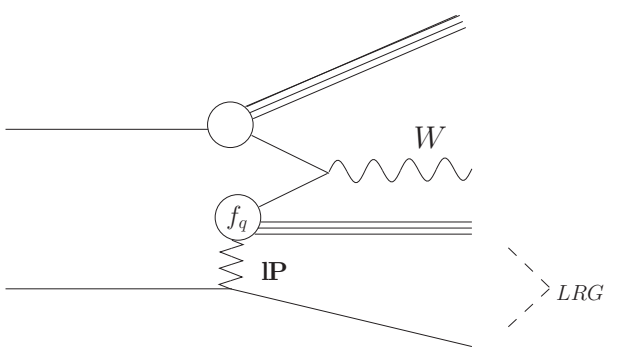

Figure 1: Sketch of the single-diffractive reaction $p p \rightarrow X p$ in which $X$ includes a $W$ boson. The symbol $\mathbb{P}$ indicates the exchange with the vacuum quantum numbers (Pomeron). The large rapidity gap (LRG) is also shown.

the CASTOR calorimeter.

\section{Monte Carlo Simulation}

Single diffractive $W$ production was simulated by using the POMWIG generator [ [], version v2.0 beta. For the diffractive PDFs and the Pomeron flux, the result of the NLO H1 2006 fit B [7] was used. A rapidity gap survival probability of 0.05 , as predicted in [8, is assumed. The cross section, for the $W \rightarrow \mu \nu$ mode, is about $70 \mathrm{pb}$, leading to $\simeq 7000$ events per $100 \mathrm{pb}^{-1}$. For non-diffractive $W$ production, the PYTHIA generator was used 9 . The cross section, for the $W \rightarrow \mu \nu$ mode, is about $22 \mathrm{nb}$ (NLO). With the given numbers for the cross sections, the ratio of diffractive to inclusive yields is $0.3 \%$. 


\section{Event Selection and Observation of SD $W$ Production}

\section{1 $W \rightarrow \mu \nu$ selection}

The selection of the events with a candidate $W$ decaying to $\mu \nu$ is the same as that used in [10. Events with a candidate muon in the pseudo-rapidity range $|\eta|>2.0$ and transverse momentum $p_{T}<25 \mathrm{GeV}$ were rejected, as were events with at least two muons with $p_{T}>20 \mathrm{GeV}$. Muon isolation was imposed by requiring $\sum p_{T}<3 \mathrm{GeV}$ in a cone with $\Delta R<0.3$. The transverse mass was required to be $M_{T}>50 \mathrm{GeV}$. The contribution from top events containing muons was reduced by rejecting events with more than 3 jets with $E_{T}>40 \mathrm{GeV}$ (selected with a cone algorithm with radius of 0.5 ) and events with acoplanarity $(\zeta=\pi-\Delta \phi)$ between the muon and the direction associated to $E_{T}^{\text {miss }}$ greater than $1 \mathrm{rad}$. Approximately 2,400 SD $W$ events and 600,000 non-diffractive $W$ events per $100 \mathrm{pb}^{-1}$ are expected to pass these cuts.

\subsection{Diffractive selection}

Figure 2 shows the generated energyweighted $\eta$ distribution for stable particles (excluding neutrinos) in diffractive and nondiffractive events, including the scattered proton; all events were generated with the scattered proton at positive rapidities (the peak at $\eta \gtrsim 10)$. Diffractive events have, on average, lower multiplicity both in the central region and in the hemisphere that contains the scattered proton, the so-called "gap side", than non-diffractive events.

The gap side was selected as that with lower energy sum in the HF. A cut was then placed on the multiplicity of tracks with $p_{T}>900 \mathrm{MeV}$ and $|\eta|<2$. For the events passing this cut, multiplicity distributions in the HF and CASTOR calorimeters in the gap side were studied, from which a diffractive sample can be extracted.

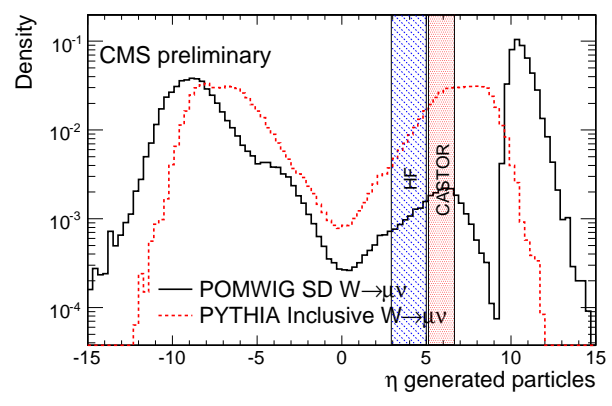

Figure 2: Generated energy-weighted $\eta$ distribution for stable particles in diffractive (POMWIG, continuous line) and non-diffractive (PYTHIA, dashed line) events. The diffractive events were generated with the gap side in the positive $\eta$ hemisphere.

\subsection{Evidence for SD $W$ Production}

\subsubsection{HF multiplicity}

Figure 3 shows the HF tower multiplicity for the low- $\eta$ ("central slice", $2.9<\eta<4.0$ ) and high- $\eta$ HF ("forward slice", $4.0<\eta<5.2$ ) regions for events with central track multiplicity $N_{\text {track }} \leq 5$. The top left and top right plots show the distributions expected for the diffractive $W$ events with generated gap in the positive and negative $Z$ direction, respectively; they exhibit a clear peak at zero multiplicity. Conversely, the non-diffractive $W$ events have on average higher multiplicities, as shown in the bottom left plot. Finally, the bottom right plot shows the sum of the POMWIG and PYTHIA distributions - this is the type of distribution expected from the data. The diffractive signal at low multiplicities is visible. 


\subsubsection{HF and CASTOR Multiplicity Distributions for the Gap Side}
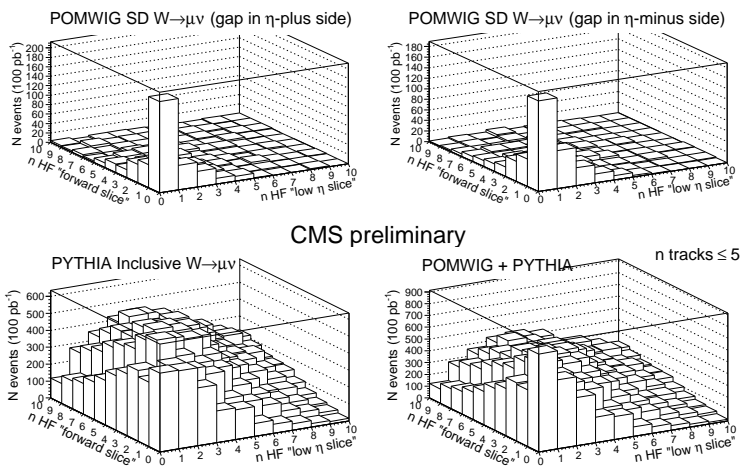

Figure 3: Low- $\eta$ ("central slice") vs high- $\eta$ ("forward slice") HF tower multiplicity distributions for events with track multiplicity in the central tracker $N_{\text {track }} \leq 5$.
The HF tower multiplicity vs CASTOR $\phi$ sector multiplicity was studied for the gap side. Since CASTOR will be installed at first on the negative side of the interaction point, only events with the gap on that side (as determined with the procedure discussed above) were considered. The CMS software chain available for this study did not include simulation/reconstruction code for CASTOR; therefore, the multiplicity of generated hadrons with energy above a $10 \mathrm{GeV}$ threshold in each of the CASTOR azimuthal sectors was used.

Figure 4 shows plots analogous to those of Fig. 3 for the combination of HF and CASTOR. The top plots show the POMwIG distributions; the few events in the top left plot are those for which the gap-side determination was incorrect. The signal to background ratio improves greatly with respect to the HF only case.

\subsection{Observation of SD $W$ Production and Extraction of a Diffractive Sample}

The two-dimensional multiplicity plots of Figs. 3 and 4 provide evidence of SD $W$ production. A simple way to isolate a sample of diffractive events from these plots is to use the zero-multiplicity bins, where the diffractive events cluster and the non-diffractive background is small.

The HF plus CASTOR condition yields the best signal to background ratio. When an integrated effective luminosity for single interactions of $100 \mathrm{pb}^{-1}$ becomes available, SD $W \rightarrow \mu \nu$ production can then be observed with $\mathcal{O}(100)$ signal events. Backgrounds other than non-diffractive $W$ production

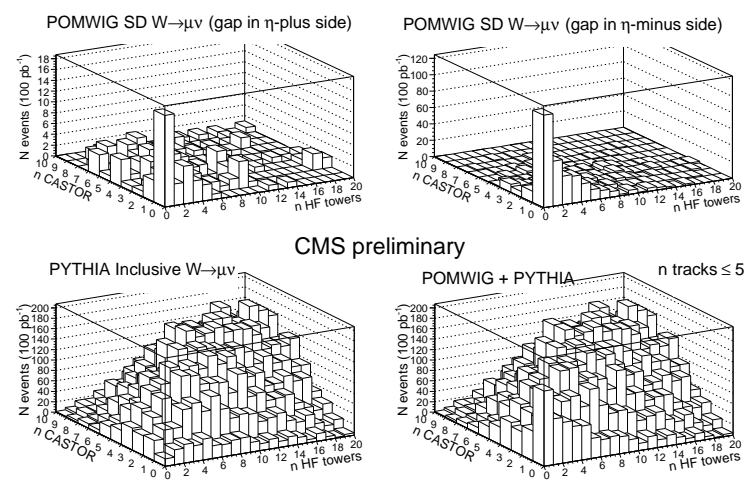

Figure 4: HF tower multiplicity vs CASTOR sector multiplicity distribution for events with track multiplicity in the central tracker $N_{\text {track }} \leq 5$.

are discussed in the next section and appear to be under control. 


\subsection{Backgrounds}

The background from QCD events has been quantified by repeating the analysis on a PYTHIA sample with lepton-enriched QCD events. The effect is to increase the number of events in the low-multiplicity region by less than $1 \%$ of the SD yield. Non-diffractive $W$ events, misidentified as diffractive, have been discussed in the previous sections, where we showed they can be kept under control.

SD $W$ production with proton-dissociation, $p p \rightarrow X N$, where $X$ contains a $W$ boson and $N$ is a low-mass state into which the proton has diffractively dissociated, is an irreducible background when $N$ escapes undetected in the forward region. A study of protondissociation has been carried out in [11, where it has been shown that about $50 \%$ of the proton-dissociative background can be rejected by vetoing events with activity in the CMS Zero Degree Calorimeter (ZDC), which provides coverage for neutral particles for $|\eta|>8.1$. The net effect is to enhance the diffractive signal in the zero multiplicity bin of Fig. 4 by about $30 \%$.

\section{Summary and Outlook}

A procedure has been discussed to arrive at the observation of single diffractive $W \rightarrow \mu \nu$ production with an integrated effective luminosity for single interactions of $100 \mathrm{pb}^{-1}$. The procedure is based on the detection of large rapidity gaps in the final state of the event using HF and CASTOR, complemented by the multiplicity information from the central tracker.

Assuming a rapidity gap survival probability of $0.05, \mathcal{O}(100)$ reconstructed signal events are expected with a high signal-to-background ratio if the CASTOR calorimeter is available. If CASTOR is not available, the HF information alone may be sufficient. Further improvements are possible if the detectors of the TOTEM experiment [12] can be used, notably the $\mathrm{T} 2$ tracker and the roman pot detectors.

\section{References}

[1] CMS Collaboration, CMS PAS DIF-07-002 (2007).

[2] J. D. Bjorken, Phys. Rev. D 47 (1993) 10111; A. B. Kaidalov et al., Eur. Phys. J. C 21 (2001) 52152.

[3] CDF Collaboration, F. Abe et al., Phys. Rev. Lett. 78 (1997) 2698.

[4] D0 Collaboration, V. M. Abazov et al., Phys. Lett. B 574 (2003) 169.

[5] See e.g.: CMS Collaboration, "CMS Physics Technical Design Report Volume I: Detector Performance and Software", CERN-LHCC 2006/001 (2006).

[6] B. E. Cox and J. R. Forshaw, Comput. Phys. Commun. 144 (2002) 104.

[7] H1 Collaboration, A. Aktas et al., Eur. Phys. J. C 48 (2006) 715.

[8] V. A. Khoze, A. D. Martin and M. G. Ryskin, Phys. Lett. B 643 (2006) 93.

[9] T. Sjostrand, S. Mrenna and P. Skands, JHEP 0605 (2006) 026.

[10] CMS Collaboration, CMS PAS EWK-07-002 (2007).

[11] CMS Collaboration, CMS PAS DIF-07-001 (2007).

[12] TOTEM Collaboration: Letter of Intent, CERN-LHCC 97-49; Technical Proposal, CERN-LHCC 99-7; Technical Design Report, CERN-LHCC-2004-002. 